\title{
TOTAL CELL NUMBER IN FETAL BRAIN
}

\author{
Grethe Badsberg Samuelsen ${ }^{1,3}$, Nenad Bogdanović ${ }^{2}$, HenNing Laursen $^{3}$, Niels \\ GRAEM $^{3}$, JøRGEN FALCK LARSEN ${ }^{4}$ AND BENTE PAKKENBERG ${ }^{1}$
}

${ }^{1}$ Neurological Research Laboratory, Kommunehospitalet, Copenhagen, Denmark; ${ }^{2}$ NEUROTEC, Geriatric Department, Karolinska Institute, Sweden; ${ }^{3}$ Laboratory of Neuropathology and Laboratory of Pediatric Pathology, Rigshospitalet, Copenhagen, Denmark; ${ }^{4}$ Department of Obstetrics and Gynaecology, Herlev University Hospital, Denmark

(Accepted December 1, 1999)

\begin{abstract}
In this study the material comprises brains from three aborted fetuses and two fullterm infants who died at birth. The gestational ages ranged from the 22nd week to term. All cases were without malformations, known chromosomal abnormality, hydrops, and systemic infections, and all had normal birth weights with fetal growth indices (observed birth weight/expected mean birth weight) between $0.9-1.05$. The preliminary results show a five fold increase in the total cell population in the marginal zone/cortical plate, MZ/CP (future neocortex), from week 22 until term. In the transient subplate zone, SP, the total cell number was more than doubled from week 22 to week 30-35, and then decreased towards term. In the intermediate zone, IZ (future white matter), the total cell population was doubled from week 22 until term. The total cell number in the ventricular/subventricular zone, VZ/SZ (germinal matrix), was reduced by a factor of five from week 22 until term. A histological differentiation between neurons and glial cells was not possible. The optical fractionator was used to estimate the total cell population in four characteristic developmental zones in the human fetal brain. Fetal brain tissue undergoes considerable and rather unpredictable shrinkage during fixation. However, using the fractionator principle it is possible to eliminate this problem, provided that the structure of interest (one brain hemisphere) is fully intact.
\end{abstract}

Keywords: fetal brain, optical fractionator, total number.

\section{INTRODUCTION}

Knowledge of the normal development of the human fetal brain is important as it may serve as a normative reference in the analysis of developmental neuroanatomy and neuropathology of the cerebrum. Stereologic counting methods have made it possible to obtain estimates on total cell numbers in human brain based on unbiased counting principles (Braendgaard et al., 1990; Jensen and Pakkenberg, 1993; Pakkenberg and Gundersen, 1997). A combination of two stereological principles, the fractionator sampling design and the optical disector, has improved the accuracy of estimates of cell numbers and the efficiency with which the estimates are obtained (West et al., 1991). A necessary prerequisite and sometimes a practical disadvantage for carrying out the fractionator principle for cell counting is that the structure of interest, one brain hemisphere, must be available and fully intact. Neither the exact volume of the region nor the exact area of their profiles need to be known, and tissue shrinkage or expansion that occurs during any stage of the tissue processing do not affect the final estimate.
We have applied the optical fractionator to five human fetal brains at mid and late gestational ages to estimate the total cell population from the inner ventricular/subventricular zone, from where all the cells derive, through the intermediate zone (prospective white matter) to the future anlage of the neocortex, the subplate and the cortical plate/marginal zone.

\section{MATERIAL AND METHODS}

\section{TISSUE}

The material comprises brains from three aborted Caucasian male fetuses age 22, 24 and 30 weeks of gestation and two fullterm (40 and 41 weeks) male infants who died at birth. The brains were obtained from necropsies and a complete autopsy including histological diagnosis and thorough neuropathological examination was carried out in each case at the university hospital Rigshospitalet, Copenhagen, Denmark. The two youngest fetuses were aborted due to chorioamnionitis, the 30 week old fetus died of acute fetal-maternal haemorrhage, and 
both fullterm infants died from acute asphyxia. One died because of tightening of a true knot of the umbilical cord, presumably 2-3 days prior to birth. The other died during labour because of an extremely short umbilical cord at breech presentation. The material was collected with the approval of the local ethical committee for Copenhagen and Frederiksberg. To ensure accurate gestational age data, we only included pregnancies for which the gestational age calculated from the last normal menstrual period agreed within \pm 7 days with an early second-trimester ultrasound determination of the fetal biparietal diameter. All fetuses were without malformations, known chromosomal abnormality, hydrops, and systemic infections, and all had normal birth weights with fetal growth indices (observed birth weight/ expected mean birth weight (Larsen et al., 1990) between 0.9-1.05.

\section{PROCESSING}

The brains were fixed in $100 \%$ concentrated formaline for a minimum of four weeks. If the brains were soft and vulnerable the formaline was exchanged with saturated picric acid for further fixation for two to four weeks. Left or right hemisphere was chosen systematically at random as far as possible, i.e. if a hemisphere was traumatised the other intact hemisphere was chosen. The hemispheres were divided into 2 to 3 blocks prior to embedding in paraffin, and then sectioned coronally with a sledge microtome using stainless steel microtome blades with a microtome setting of $40 \mu \mathrm{m}$. The blocks were cut serially and exhaustively throughout the hemisphere. Depending on the brain size, a certain fraction of the sections was sampled systematically and randomly. The sections were mounted on double silane coated glass slides and immediately dried at $40^{\circ} \mathrm{C}$ for 24 hours. Prior to staining, the sections were first heated to $60^{\circ} \mathrm{C}$ for 30 minutes, then dehydrated in xylene for 45 minutes, followed by graded series of ethanol solutions $(15 \mathrm{~min}$ in $99 \%$ alcohol, $10 \mathrm{~min}$ in $96 \%$ alcohol, $5 \mathrm{~min}$ in $70 \%$ alcohol), and $5 \mathrm{~min}$ in distilled water. Eventually, the sections were stained for $45 \mathrm{~min}$ with a modified Giemsa staining containing: $25 \mathrm{ml}$ Giemsa stain stock solution (Merck, Germany, product 1.09204), and $250 \mathrm{ml}$ potassium-hydrogen-phosphate, $\mathrm{pH} 4.5$ - being filtered prior to use. Finally, the sections were differentiated through $96 \%$ ethanol solution in 1-5 min, $99 \%$ ethanol solution in 5-10 min, and in xylene for 15 min The distance between the upper and lower surfaces of the sections was measured with a Heidenhain microcator attached to the microscope stage using a $100 x$ oil objective. A delineation of the developmental zones was done according to histological criteria (Kostovic and Rakic, 1990): ventricular/ subventricular zone (VZ/SZ), intermediate zone (IZ), subplate (SP) and marginal zone/cortical plate $(\mathrm{MZ} / \mathrm{CP})$.

\section{THE FRACTIONATOR SAMPLING SCHEME}

The optical fractionator was used to estimate the total number of cells in the MZ/CP, SP, IZ and VZ/SZ in the brains on $40-\mu \mathrm{m}$-thick Giemsa stained paraffin sections. A sampling fraction (ssf) between $1 / 80$ th or $1 / 200$ th section was chosen, depending on the size of the brain, in order to achieve approximately 10-16 sections per brain using a systematic random sampling design. The cells were counted directly in a known fraction of each zone using the optical disector in a systematic, random pattern. The area of the counting frame of the disector, a(frame), was known relative to the area associated with each movement in the $\mathrm{x}$-y-direction (asf). The height (h) of the disector was chosen to be $10 \mu \mathrm{m}$ and the mean section thickness (t) in each brain was estimated from measurements of the thickness of the sections made at every 5 th disector.

The total number of cells was then calculated as

$$
N=\sum Q^{-} * \frac{t}{h} * \frac{1}{s s f} * \frac{1}{a s f}
$$

where $\sum \mathrm{Q}^{-}$is the total number of cells counted in all disectors.

\section{STATISTICS}

The Spearman correlation coefficient was used to correlate the total number of cells and gestational age (Fig. 1).

\section{RESULTS}

As illustrated in the figure, the total cell population was increased by a factor of five in the marginal zone/cortical plate, MZ/CP (future 6-layers cortex), from $5.7 \times 10^{9}$ cells in week 22 to $31.1 \times 10^{9}$ cells in week $41\left(\mathrm{R}^{2}=0.98\right)$.

In the intermediate zone, IZ (future white matter) the total cell population was doubled from $2.0 \times 10^{9}$ cells in week 22 to $4.4 \times 10^{9}$ cells at term $\left(\mathrm{R}^{2}=0.95\right)$.

The total cell number in the ventricular/ subventricular zone, VZ/SZ (germinal matrix), was reduced by a factor of five, from $5.1 \times 10^{9}$ cells in week 22 to approximately $1.1 \times 10^{9}$ cells at term $\left(\mathrm{R}^{2}=0.97\right)$. 


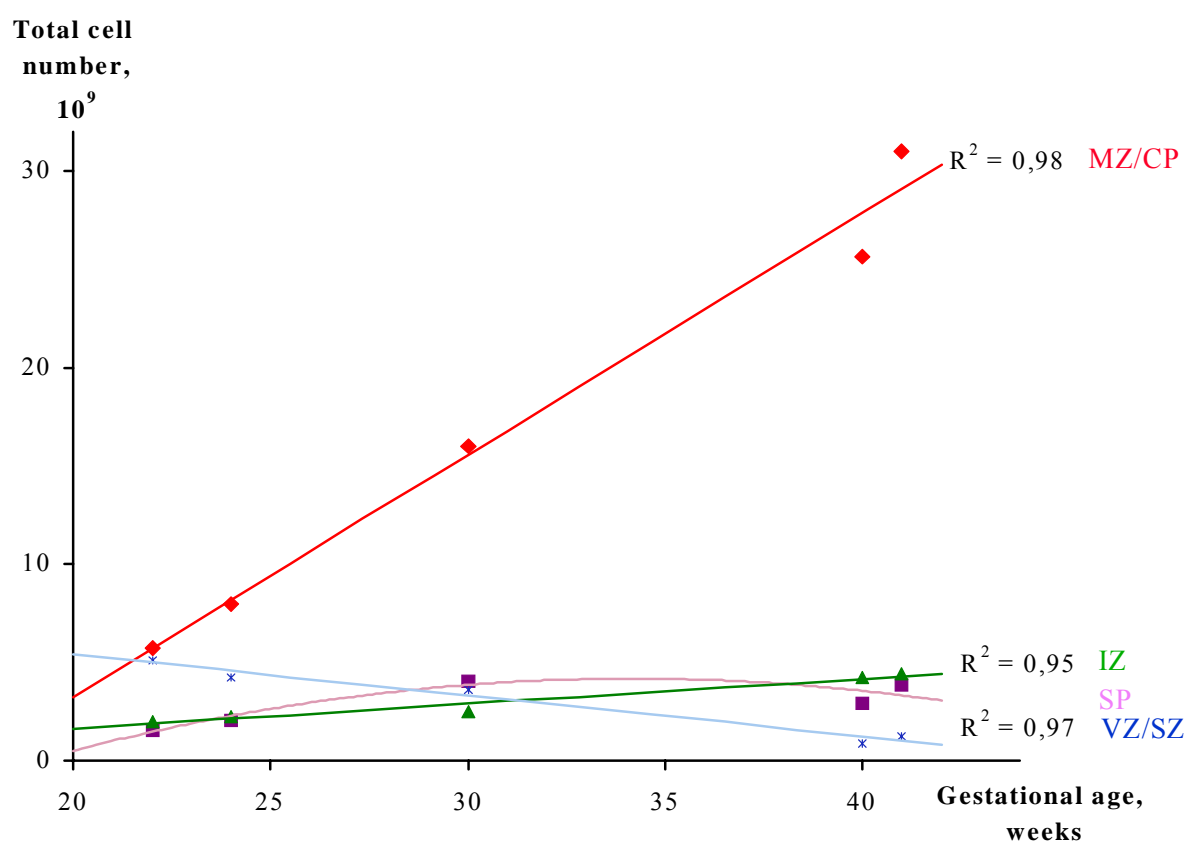

Fig. 1. The total number of cells $\left(10^{9}\right)$ is shown in relation to gestational age (weeks) in the different zones. ४: marginal zone and cortical plate, $\mathbf{\square}$ : subplate, $\mathbf{\Delta}$ : intermedia zone, $\mathrm{x}$ : ventricular and subventricular zones.

\section{DISCUSSION}

During fetal life the human brain undergoes various cellular processes which contribute to shape and define cell fate in the brain. These processes include cellular proliferation, migration, differentiation, synaptogenesis and cell death, and take place in characteristic developmental zones (Kostovic and Judas, 1998). Cell density and cell number vary from zone to zone depending on the sequencing of developmental events. Previous quantitative morphometric studies on human fetal brain have quantified total cell/ neuron numbers. However, these studies were mostly based on 2D sections, reporting cell density (Shankle et al., 1999; Rabinowicz et al., 1996; Landing et al., 1998), a parameter that is very difficult to interpret in fetal brain tissue, as the brain undergoes considerable and rather unpredictable shrinkage during preparation. One study, however, published in abstract form (Ansari et al., 1995), has estimated total cell numbers stereologically in postnatally compromised infants and SIDS cases from 3 weeks to 1 year of age. This study reported a total number of neocortical cells of $101 \times 10^{9}, 3$ weeks postnatally, more than 3 times our estimates in the fullborn cases. The design of their study was based on numerical density multiplied with the neocortical volume estimated from Cavalieri's principle. The reason for the difference in total cell number in their study compared to our results is yet unknown.

In 1973 Dobbing et al. quantified DNA content in the developing human fetal brain. They analysed 35 fetal brains at different gestational ages. Assuming the majority of cells is diploid and has a constant amount of DNA per diploid nucleus, Dobbing et al. interpreted $\mathrm{DNA} / \mathrm{g}$ tissue as an indirect measurement of "cellularity", and total amount of DNA as an indirect measurement of total cell number. However, they knew that this "cellularity" is a difficult parameter to interpret in the growing brain, because the "cellularity" decreases with increasing gestational age at the very time when the total cell number is rising. This decrease in "cellularity" is due to several other parameters of growth which have a net diluting effect on the total cell number. Thus the growth in weight outpaces the growth in numbers.

To differentiate between neurons and glial cells in fetal brain is a major problem. Especially in the younger fetuses, where the immature cells do not yet express specific neuronal/glial markers. Furthermore the fetal tissue is loose and very fragile and endures epitope retrieval procedures badly. We tried in the two fullborn cases to differentiate between neurons and glial cells using histological definitions. In the cortical plate the neuronal pyramidal neurons in the layers III and $\mathrm{V}$ were easy to distinguish, but it was still not possible to differentiate between the smaller neurons and glial cells. Thus our results represent the total cell number in the particular zones.

The total cell population in the cortical plate at term is estimated to approximately $30.0 \times 10^{9}$ cells in our study, whereas in the adult human brain (age $80 \mathrm{yr}$ ) the total cell population is $47 \times 10^{9}$ cells with a glial 
cell/neuron ratio of 1.5 (personal communication, Pelvig, 1999). Most probably this large difference is due to an increase of neocortical glial cells which is in accordance with an intensive production of glial cells taking place pre- and postnatally (Schmechel and Rakic, 1979).

In the transient subplate zone, a part of the future 6layers cortex, it is interesting that the total cell number was more than doubled from $1.6 \times 10^{9}$ cells in week 22 to $4.0 \times 10^{9}$ cells in week 30 , and after week 35 decreased towards term.

The design of this human developmental study differs considerably from that of animal studies. For animals, the precise gestational age and the size of groups with a particular age can be planned, and thus the inter-individual animal variation at the different prenatal ages can be estimated as well. This is not possible in human studies where we are dealing with limitations in the availability of human tissue and dating of the gestational ages. In the present study we have overcome most technical problems by applying the optical fractionator on human fetal brain tissue. Future quantitative studies could focus on differentiation between different cell types using specific staining methods and cellular markers.

\section{ACKNOWLEDGEMENT}

This study was supported by The Ville Heise's Grant, The Cripple Foundation in Denmark (Vanførefonden), Danish Hospital Foundation for Medical Research, Region of Copenhagen, The Faeroe Islands and Greenland, The Danish Medical Association Research Fund, Financial Support for the Encouragement of Clinical Research, University of Copenhagen, and Gun and Bertil Stohne Stiftelsen.

\section{ETHICS}

Jr. nr. (KF) 01-247/95.

A preliminary report of some of the data (Badsberg Samuelsen et al., 1999) has been presented at the $\mathrm{X}^{\text {th }}$ International Congress for Stereology, Melbourne, Australia, 1-4 November 1999.

\section{REFERENCES}

Ansari T, Yan L, Howard CV, Sibbons PD, Macmillan A, Van Velzen D (1995). A stereological study investigating the total number of neocortical neurons in postnatally compromiseed humans and SIDS cases from 3 weeks to 1 year of age. $9^{\text {th }}$ International Congress for Stereology. Copenhagen.

Braendgaard H, Evans SM, Howard CV, Gundersen HJG (1990). The total number of neurons in the human neocortex unbiasedly estimated using optical disectors. J Microsc 157:285-304.

Dobbing J, Sands J (1973). Quantitative growth and development of human brain. Archives of Disease in Childhood 48: 757-67.

Jensen GB, Pakkenberg B (1993). Do alcoholics drink their neurons away? Lancet 342:1201-4.

Kostovic I, Rakic P (1990). Developmental history of the transient subplate zone in the visual and somatosensory cortex of the macaque monkey and human brain. J Comp Neurol 297: 441-70.

Landing BH, Shankle WR, Hara J (1998). Constructing the human cerebral cortex during infancy and childhood: types and numbers of cortical columns and numbers of neurons in such columns at different age-points. Acta Paediatr Jpn 40:530-43.

Larsen T, Petersen S, Greisen G, Larsen JF (1990). Normal fetal growth evaluated by longitudinal ultrasound examinations. Early Hum Dev 24:37-45.

Pakkenberg B, Gundersen HJG (1997). Neocortical neuron number in humans: effect of sex and age. J Comp Neurol 384:312-20.

Rabinowicz T, de Courten-Myers GM, Petetot JM, Xi G, de los Reyes E (1996). Human cortex development: estimates of neuronal numbers indicate major loss late during gestation. J Neuropathol Exp Neurol 55:320-8.

Schmechel DE, Rakic PA (1979). Golgi study of radial glial cells in developing monkey telencephalon: morphogenesis and transformation into astrocytes. Anat Embryol 156:115-52.

Shankle WR, Rafii MS, Landing BH, Fallon JH (1999). Approximate doubling of numbers of neurons in postnatal human cerebral cortex and in 35 specific cytoarchitectural areas from birth to 72 months. Pediatr Dev Pathol 2:244-59.

West MJ, Slomianka L, Gundersen HJG (1991). Unbiased stereological estimation of the total number of neurons in the subdivisions of the rat hippocampus using the optical fractionator. Anat Rec 231:482-97. 